Received: 2015.06 .18
Accepted: 2015.06 .28
Published: 2015.09 .29
Authors' Contribution:
A Study Design
B Data Collection
C Statistical Analysis
D Data Interpretation
E Manuscript Preparation
F Literature Search
G Funds Collection

\section{Synovial Hemangioma of the Knee Joint:} Magnetic Resonance Imaging Findings

\author{
Ibrahim Guler ${ }^{\text {AFF }}$, Alaaddin Nayman ${ }^{\text {BED }}$, Mustafa Koplay ${ }^{\text {GF }}$, Yahya Paksoy ${ }^{\text {AB }}$ \\ Department of Radiology, Selcuk University Faculty of Medicine, Konya, Turkey \\ Author's address: Ibrahim Guler, Department of Radiology, Selcuk University Faculty of Medicine, Konya, Turkey, \\ e-mail: dribrahimguler@gmail.com
}

\title{
Summary
}

Background: Synovial hemangioma is benign tumor of the joints and is seen relatively rare. The most affected joint is knee but should also be seen in other joints. The disease is usually symptomatic. They are classified as juxta-articular haemangioma, intra-articular haemangioma or an intermediate type of hemangioma with intra- and extraarticular components.

Case Report: $\quad$ A 19-years-old male patient presented with swollen and painful knee. The laboratory findings and physical examination were normal. MRI demonstrated a large lesion that was filling the suprapatellar bursa.

Conclusions: All radiologic examinations should be used in diagnosis but magnetic resonance imaging is the noninvasive method and excellent modality in the evaluation of soft tissues. In this paper, a 19-yearold male patient with the diagnosis of synovial hemangioma is reported and its radiologic findings are mentioned.

\section{MeSH Keywords: $\quad$ Bursa, Synovial • Hemangioma • Magnetic Resonance Imaging \\ PDF file: $\quad$ http://www.polradiol.com/abstract/index/idArt/895060}

\section{Background}

Synovial hemangioma is a rare benign tumour of the joints and is located in the knee in most patient [1]. The disease affects children and young adults. It is usually symptomatic and the mostly seen symtoms are pain and swelling. The lesion is frequently misdiagnosed, leading to a delay in diagnosis. We reported a case of synovial hemangioma in the knee joint which was diagnosed with magnetic resonance imaging (MRI).

\section{Case Report}

A 19-year-old male patient presented with a swollen and painful knee. The laboratory findings and physical examination were normal, except for the tenderness and swelling in the anterior knee.The knee MRI (Siemens, Aera, Erlangen, Germany) was performed. A routine knee sequence including proton density imaging in 3 orthogonal planes, coronal T2-weighted image with fat saturation were obtained. Precontrast and postcontrast Tl-weighted images were also included. MRI demonstrated a large lesion that was filling the suprapatellar bursa. The lesion was extending to the surrounding muscles on and no bone involvement was seen. The lesion was hyperintense related to the muscles and including hypointense linear structures within the center on proton density and T2-weighted fat saturated images (Figure 1A, 1B). The lesion was isointense to the muscle on precontrast Tl-weighted images and demonstrated heterogenous marked enhancement after contrast administration (Figure 1C, 1D).

\section{Discussion}

Synovial hemangiomas are benign knee tumours originating in the subsynovial mesenchyma of the synovial membrane. They are classified as juxta-articular haemangioma, intra-articular haemangioma or an intermediate type of hemangioma with intra- and extraarticular components [2]. Synovial hemangiomas constitute less than $1 \%$ of all haemangiomas and are most common in the knee, but have also been reported in other joints, including the elbow, ankle and wrist [3].

The clinical features include the pain and swelling in almost all patients. The tenderness, limitation of knee, 


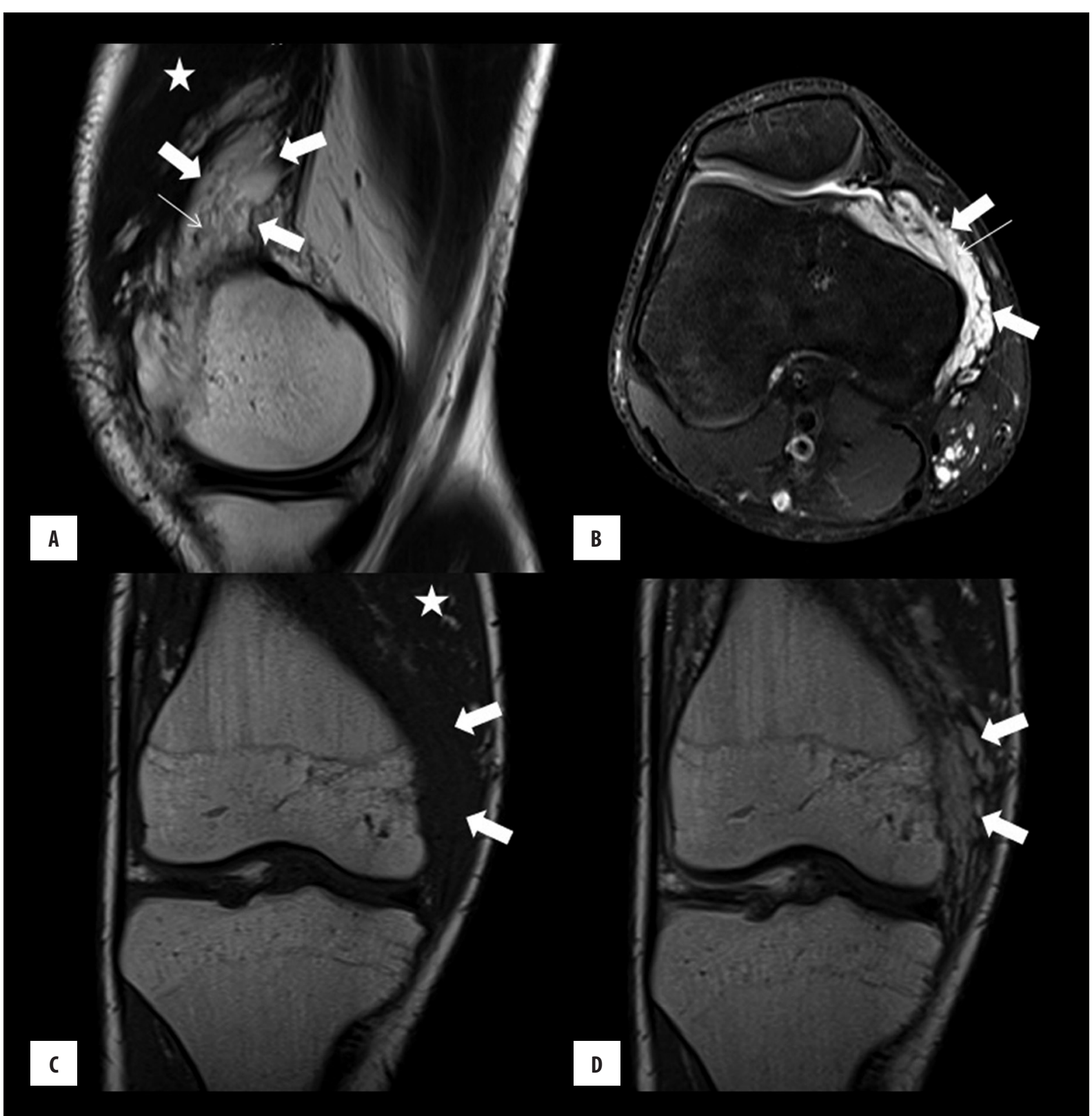

Figure 1. The lesion (thick arrows) was hyperintense related to the muscles (asteriks) and included hypointense linear structures (thin arrow) within the center on sagittal T2-weighted (A) and axial proton density fat-saturated images (B). The lesion (arrows) was isointense to the muscles (asteriks) on precontrast coronal T1-weighted images (C) and demonstrated heterogenous marked enhancement after contrast administration (arrows) (D).

quadriceps atrophy, joint effusions (hemarthrosis) are the other symptoms of the synovial hemangiomas. It can mimic the meniscal pathologies. Especially in patients with a history of recurrent pain and swelling with hemarthrosis, synovial hemangioma should be considered in the diagnosis $[4,5]$.

Plain radiographies, gray-scale and color doppler ultrasonography (US), angiography and computed tomography or MRI can be used in the diagnosis of synovial hemangiomas. Plain radiographs are not diagnostic in half of the patients and could show the effusions and soft tissue masses. A periosteal reaction, phelobolits, and bone erosions are also identified with plain radiographs. Ultrasonographic examinations show the vascular structures within the lesion and are helpful in the differentation of cystic lesions from the solid ones. Computed tomography may demonstrate the lesion but this method is not specific in the diagnosis and especially in small lesions it is not able to differantiate the lesion from normal structures. Also, the radiation risk is another disadvantage. Angiography is an invasive method and should to be the initial procedure in the diagnostics. It is effective in showing the extent of the lesion and the vascular origin. The MRI is the best modality in evaluating soft tissues and should be considered as a diagnostic method. Synovial hemangiomas appear as intermediate signal intensity on Tl-weighted images, isointense or slightly hyperintense than surrounding muscles. 
The lesion appears hyperintense on T2-weighted and fatsupressed images. Thin, serpentine and low-intensity septa are also detected on T2-weighted and fat-supressed images. After contrast administration, the lesion shows marked enhancement. The MRI is also used to differentiate the lesion from other pathologies [2,6-10].

The differential diagnosis should include mainly pigmented villonodular synovitis, synovial sarcoma, arthropathies (rheumatoid arthritis, hemophilic arthropathy, synovial osteochondromatosis or lipoma arborescens) usually being distinguished clinically or after MRI interpretation $[8,9,11]$.

\section{Conclusions}

In conclusion, especially in patients with a history of recurrent pain and swelling, synovial hemangioma should be considered in the differential diagnosis. In such patients, radiological evaluation with MRI is diagnostic almost in all patients and should be used as an initial procedure.

\section{Conflict of interest}

The authors declare that they have no conflict of interest.

\section{References:}

1. Greenspan A, Azouz EM, Matthews J II, Decarie JC: Synovial haemangioma: imaging features in eight histologically proven cases, review of the literature, and differential diagnosis. Skeletal Radiol, 1995; 24: 583-90

2. Silit E, Mutlu H, Pekkafali $\mathrm{Z}$ et al: Synovial hemangioma of the knee invading the femur. Skeletal Radiol, 2002; 31: 612-14

3. Su JT, Cheon SJ, Choi SJ: Synovial Hemangioma of the Knee. Arthroscopy, 2003; 19: 27-30

4. Sasho T, Nakagawa K, Matsuki $\mathrm{K}$ et al: Two cases of synovial haemangioma of the knee joint: Gd-enhanced image features on MRI and arthroscopic excision. Knee, 2011; 18: 509-11

5. Coulier B, Mailleux P: Synovial hemangioma diagnosed during knee arthro-CT. JBR-BTR, 2004; 87: 183-85

6. Narvaez JA, Narváez J, Aguilera C et al: MR imaging of synovial tumors and tumor-like lesions. Eur Radiol, 2001; 11: 2549-60
7. Vakil-Adli A, Zandieh S, Hochreiter J et al: Synovial hemangioma of the knee joint in a 12-year old boy: a case report. J Med Case Rep, 2010; 4: 105

8. De Gori M, Galasso O, Gasparini G: Synovial hemangioma and osteoarthritis of the knee: a case report. Acta Orthop Traumatol Turc, 2014; 48: 607-10

9. De Filippo M, Rovani C, Sudberry JJ et al: Magnetic resonance imaging comparison of intra-articular cavernous synovial hemangioma and cystic synovial hyperplasia of the knee. Acta Radiol, 2006; 47: 581-84

10. Holzapfel BM, Geitner U, Diebold J et al: Synovial hemangioma of the knee joint with cystic invasion of the femur: A case report and review of the literature. Arch Orthop Trauma Surg, 2009; 129: $143-48$

11. Tzurbakis M, Mouzopoulos G, Morakis E et al: Intra-articular knee haemangioma originating from the anterior cruciate ligament: a case report. J Med Case Rep, 2008; 2: 254 